\title{
Correction to: Reviving Sudan's Ancient History and Tourism
}

Alaa Abbas, Fatimah Abbas, and Aida Nayer

\section{Correction to: \\ Chapter "Reviving Sudan's Ancient History and Tourism" in: \\ D. Hawkes et al. (eds.), Conservation of Architectural Heritage, Advances in Science, Technology \& Innovation, https://doi.org/10.1007/978-3-030-10871-7_11}

The original version of this chapter "Reviving Sudan's Ancient History and Tourism" was inadvertently published with incorrect authorship. The corresponding author and the sequence has been corrected in the chapter. The erratum chapter and the book have been updated. 